\title{
Mobile Arbeit: zwischen Autonomie und Fremdbestimmung
}

MANUELA MASCHKE, GERD NIES, GERLINDE VOGL

Mobile Arbeit ist räumlich entgrenzte Arbeit. Es gibt sie in unterschiedlichen Formen: Arbeit zu Hause, beim Kunden, im Zug oder anderswo. Mobil ist Arbeit nicht nur aufgrund von vielen Dienstreisen, sondern auch bei mobilen Arbeitsinhalten wie z. B. in virtuellen Projektteams. Relevante Handlungsfelder für betriebliche Interessenvertretungen sind: Arbeitszeit, ständige Erreichbarkeit, Gesundheitsschutz und Kompetenzentwicklung. Dies ergab eine Auswertung von 96 betrieblichen Vereinbarungen und ergänzenden Gesprächen/ Fallstudien mit betrieblichen Experten. ${ }^{1}$

Mobile Endgeräte wie z. B. Smartphones machen Datentransfer und globale Kommunikation einfach. Bestimmte Arbeiten lassen sich so im Prinzip jederzeit und überall erledigen. Das ist ihr Vorteil und zugleich auch ihr Nachteil, denn die Grenzen zwischen dem Arbeitsort, der Arbeitszeit und dem Leben in- und außerhalb der Arbeitswelt verschwimmen noch stärker.

Ein weiterer aktuell wichtiger Trend verbirgt sich hinter dem Begriff der Digitalisierung: Mehr Arbeit wird nicht nur mit mobilen Arbeitsgeräten erledigt, sondern zunehmend sind die Arbeitsgegenstände selbst digitalisiert bzw. vernetzt. ${ }^{2}$ Die Computernutzung am Arbeitsplatz liegt zwischen 63 und 98 \%, je nach Branche. Neue Produktions- und Arbeitsorganisationen bestehen aus vernetzten Systemen, Objekten und Umgebungen (Industrie 4.0). Produkte werden sozusagen digital veredelt; sie werden „schlau“. Das gilt auch für Energieversorgung, Gesundheitsdienstleistungen etc. Zusätzlich verbinden sich mobile Endgeräte und Cloud-Infrastrukturen, sodass der stationäre PC langsam aber sicher ins Museum wandert. ${ }^{3}$ Damit wachsen die Möglichkeiten, den Arbeitsort flexibel zu wählen. Weil der Arbeitsplatz nicht mehr starr, sondern beweglich ist, können individuelle Bedürfnisse berücksichtigt werden. „Smart Working Center“ in Wohnortnähe entstehen, man arbeitet in öffentlichen Cafés, TransitLounges an Verkehrsknotenpunkten, in Verkehrsmitteln, Wohnungen von Freunden und Angehörigen oder im Arbeitsurlaub. $^{4}$

Die Bandbreite mobiler Arbeit reicht von der Teleheimarbeit über virtuelle Projektteams, die räumlich getrennt an gemeinsamen Projekten arbeiten, bis hin zu Arbeit beim Kunden, wenn z. B. Ingenieure weltweit Anlagen warten, installieren bzw. in Betrieb nehmen. Mobilitätsformen lassen sich danach unterscheiden, was bzw. wer mobil ist. Zum einen kann es die Person sein, die räumlich mobil ist, die Arbeit an unterschiedlichen Orten ausführt, oder es sind die Arbeitsinhalte, die per Datenleitung mobil sind. Letzteres wird meist als virtuelle Mobilität bezeichnet. Seit aber auch Datennetze überall zugänglich sind, Geräte nicht mehr stationär verbunden sein müssen, lassen sich diese Formen der Mobilität vielfältig verbinden: Sowohl die Personen als auch die Arbeitsinhalte werden mobil. Hier spricht man von mobiler Telearbeit - die Ausbreitung dieser Arbeitsform kann man seit Jahren in wachsendem Umfange im Zug, in der Hotellobby oder in Cafés beobachten. Arbeiten „anytime and anywhere“ wird zum Angebot und zur Drohung gleichermaßen. Denn in der Praxis ergeben sich diverse Einschränkungen: technologische (fehlerhaftes bzw. falsches Equipment), praktische (logistische Probleme beim Transport von benötigtem Material) und soziale (kulturelle Normen über Anwesenheit). Sie behindern nicht selten orts- und zeitunabhängiges Arbeiten. ${ }^{5}$

Die einzelnen Formen mobiler Arbeit treten oftmals nicht isoliert auf, sondern werden je nach Tätigkeit auch untereinander kombiniert. Ein IT-Berater arbeitet beispielsweise über einen längeren Zeitraum hinweg beim Kunden, auf dem Weg zum Kunden arbeitet er im Zug oder im Hotel und nach einem Einsatz beim Kunden zu Hause im Home-Office, um endlich in Ruhe die notwendigen Berichte verfassen zu können. Die Anforderungen an Beschäftigte sind nach Art der mobilen Arbeit sehr unterschiedlich: Teleheimarbeiter verfügen über keine räumliche Trennung zwischen Arbeit und Leben. Diese müssen sie in der heimischen Wohnung selbst herstellen. Bezogen auf Familie muss trotz Anwesenheit Abwesenheit hergestellt werden, während ein mobiler Arbeitnehmer, der die ganze Woche beim Kunden ist, mittels mobiler Technik Anwesenheit bei körperlicher Abwesenheit herstellen muss.

1 Vogl, G./Nies, G. (2013): Mobile Arbeit, Frankfurt a. M.

2 Schwemmle, M./Wedde, P. (2012): Digitale Arbeit in Deutschland, Bonn.

3 Arbeitskreis Industrie 4.0 (2012): Abschlussbericht, Berlin.

4 Spath, D. (2012): Arbeitswelten 4.0, Stuttgart.

5 Wiberg, M./Ljungberg, F. (2001): Exploring the vision of anytime, "anywhere" in the context of mobile work, in: Malhotra, Y.: Knowledge management and business model innovation, Hershey, S. 153-165. 
Profitiert der alternierend Arbeitende davon, weniger pendeln zu müssen, wird der reisende Beschäftigte mit Verspätungen, Staus und mit vollen Zügen konfrontiert.

Es entsteht ein etwas bitterer Vorgeschmack auf die $\mathrm{Zu}$ kunft, wenn man aktuelle Untersuchungen und Beiträge zum Thema Gesundheit und Flexibilität zur Kenntnis nimmt: wachsender Stress durch zu viel Hetze, zu viel Komplexität, zu viel Arbeit. ${ }^{6}$ Es entstehen zwar Entscheidungsspielräume für Beschäftigte, zugleich wachsen Leistungserwartungen und Anforderungen. Arbeit wird noch intensiver. ${ }^{7}$

Die Quadratur des Kreises aus unserer Sicht lautet: Es gilt, sowohl individuelle Freiräume zur Gestaltung von Arbeitszeit, -ort und -organisation zu erhalten wie auch Schutz vor einem Übermaß an Entgrenzung, Flexibilisierung und Arbeitsverdichtung zu finden. Als zentrale Handlungsfelder bei der Gestaltung mobiler Arbeit haben sich Arbeitszeit und Arbeitsüberlastung, verbunden mit ständiger Erreichbarkeit, Gesundheitsschutz, Kompetenzentwicklung und Personalpolitik herauskristallisiert.

\section{Handlungsfeld Arbeitszeit}

Bei mobiler Telearbeit und Teleheimarbeit wird häufig auf jede Form der Zeiterfassung verzichtet. Was scheinbar größere Freiheit bei der Zeitgestaltung bedeutet, führt in Verbindung mit der Erwartung, ständig erreichbar zu sein, zu erhöhtem Arbeits- und Zeitdruck.

Wird beim Kunden gearbeitet, gelten die Arbeitszeiten des (Entsende)Betriebs nicht mehr. Natürlich finden Arbeitszeitregelungen noch Anwendung, Zeiten werden in Zeitkonten eingetragen. Aber die Zeiten werden in den meisten Fällen vergütet und nur in seltenen Fällen ausgeglichen. Allerdings wird die Arbeitszeit häufig bei zehn Stunden „abgeschnitten“, damit wenigstens auf dem Papier die Grenzen des Arbeitszeitgesetzes (ArbZG) beachtet werden.

Ein besonderes Problemfeld ist die Kombination von Arbeits- und Reisezeiten. Die zeitliche Inanspruchnahme ist oft besonders hoch, aber eine volle Anrechnung von Reisezeiten auf Arbeitszeiten findet nicht statt. Reisezeit geht auf Kosten der privaten Lebenszeit. In den ausgewerteten Vereinbarungen geht die Bewertung von Reisezeit weit auseinander, die Möglichkeit zur Abgeltung in Freizeit ist stark eingeschränkt. Bei der Frage, wann Reisezeit als Arbeitszeit zu werten ist, wird bislang nur unzureichend berücksichtigt, dass heute auf Reisen zunehmend gearbeitet wird. Diese Schwierigkeiten spiegeln sich auch in der Beteiligung des Betriebsrats wider: Bis heute gilt in der Regel, dass die Anordnung von Dienstreisen ebenso mitbestimmungsfrei gehandhabt wird wie die Festlegung der Reisezeiten. Dabei bieten die Mitbestimmung zu Versetzung und Auswahlrichtlinien und die Mitbestimmung bei der Festlegung der Arbeitszeiten durchaus (ungenutzte) Möglichkeiten der Gestaltung. Extreme Arbeitszeitbelastungen zeigen sich bei Montageeinsätzen. Die Einhaltung von vertraglichen und gesetzlichen Arbeitszeitregelungen wird hier überlagert von den tatsächlichen Anforderungen vor Ort. Die oft extremen Anforderungen korrespondieren mit einem durchgehend beklagten Personalmangel. Das führt zu hohen Zeitguthaben in einem Fall berichteten Betriebsrat und Personalleitung von Guthaben einzelner Monteure von mehreren tausend Stunden -, die aber nur selten zumindest auch teilweise in Zeit ausgeglichen werden können. Nur vereinzelt sind personalpolitische Ansätze erkennbar, die darauf abzielen, Belastungen im Rahmen einer Personalentwicklung und -steuerung anders $\mathrm{zu}$ verteilen und damit auch zu reduzieren. Gute Regelungen in Betriebsvereinbarungen, z. B. über einen Personalschlüssel und Neueinstellungen abhängig von prognostizierten und angefallenen Mehrarbeitsstunden, bleiben wirkungslos, weil geeignete Bewerber auf dem Arbeitsmarkt nicht gefunden werden.

Häufig sind Personalmangel und der Einsatz der immer gleichen Beschäftigten mit dafür verantwortlich, dass ein Zeitausgleich für angefallene Reise- und Arbeitszeit nicht möglich ist. Verbindliche Regelungen zum Zeitausgleich sind zwar notwendig, aber nicht ausreichend; sie müssen durch Einstellungsklauseln und durch Personalentwicklungsmaßnahmen ergänzt werden, die darauf abzielen, mobile Arbeit breiter zu verteilen. Lediglich in einer unserer Fallstudien (vgl. Fußnote 1) konnte von solchen Maßnahmen berichtet werden: Hier wurde, wenn auch nur in einer Abteilung, gezielt der Kreis der Beschäftigten erweitert, die zu solchen Einsätzen herangezogen werden können, um Einsatzhäufigkeit und -dauer zu begrenzen.

\section{Handlungsfeld ständige Erreichbarkeit}

Wenn die Technik Erreichbarkeit ermöglicht, dann wird Erreichbarkeit auch erwartet. Rund $70 \%$ von 854 befragten Unternehmen erwarten von ihren Beschäftigten Erreichbarkeit auch außerhalb der betrieblichen Anwesenheit oder Arbeitszeit. ${ }^{8}$ Es entstehen Konflikte zwischen Mitarbeitern und Vorgesetzten oder unter Beschäftigten, auch wenn nach der Betriebsvereinbarung niemand verpflichtet ist, außerhalb der Arbeitszeit erreichbar zu sein. Dass ständige Erreichbarkeit zu einer Fehlbelastung wird, zeigt die Untersuchung des Deutschen Gewerkschaftsbundes (DGB) zu guter Arbeit: „Je mehr von den Beschäftigten Erreichbarkeit verlangt wird, desto größer ist auch der Anteil der extrem Gehetzten. ${ }^{\text {"9 }} 21$ \% der Beschäftigten fühlen sich in ihrer Arbeit gehetzt, bei

6 Badura, B./Ducki, A./Schröder, H./Klose, J./Meyer, M. (2012): Fehlzeitenreport, Heidelberg.

7 Hauff, S./Kirchner, S. (2013): Wandel der Arbeitsqualität, in: Zeitschrift für Soziologie 42 (4), S. 337-355.

8 Bitkom (2013): Arbeit 3.0, Arbeiten in der digitalen Welt, Berlin.

9 DGB-Index Gute Arbeit (2012): Arbeitshetze - Arbeitsintensivierung - Entgrenzung, Repräsentativbefragung 2011, Berlin. 
denjenigen, die ständig erreichbar sind, sind es mit $38 \%$ fast doppelt so viele. ${ }^{10}$ Mehrere Studien bestätigen negative $\mathrm{Zu}$ sammenhänge. ${ }^{11}$ Manche Vereinbarungen begrenzen den Zugriff aufs Firmennetz auf die Zeitspanne der betrieblichen Gleitzeit. Andere regeln, dass die Beschäftigten außerhalb ihrer individuellen Arbeitszeit nicht erreichbar sein müssen oder ergänzen dies um die ausdrückliche Verpflichtung für Vorgesetzte, einer Erwartungshaltung entgegenzuwirken, die eine ständige Erreichbarkeit der Beschäftigten voraussetzt. Klare Vertretungsregelungen für den E-Mail-Verkehr bei Fehlzeiten sowie bezahlte, geplante Rufbereitschaften mit Sonderurlauben sind keine neuen, aber inzwischen etablierte Verfahren.

Im Rahmen der Fallstudien äußerten Beschäftigte und Betriebsräte jedoch auch erhebliche Vorbehalte gegen eine rein „technische“ Lösung des Problems. Sie wird als Bevormundung empfunden und kann den Arbeitsstress sogar noch erhöhen. Das ist plausibel: Wenn nicht mehr die geschuldete Arbeitszeit, sondern die Einhaltung des Projekttermins die Arbeit bestimmt, kann die Verweigerung oder Einschränkung der Kommunikationsmöglichkeit den Druck erhöhen und Stress verursachen. Einfache Lösungen zur Regelung von Erreichbarkeit gibt es nicht. Es wird notwendig, den Umgang mit dieser Bereitschaftszeit zu regeln und durchzusetzen, dass Erreichbarkeit außerhalb der individuellen Arbeitszeit auch Arbeitszeit ist und verbucht werden muss, wenn sie in Anspruch genommen wird.

\section{Handlungsfeld Gesundheitsschutz}

Die Verbindung von mobiler Arbeit und Gesundheitsschutz ist bislang kaum ein Thema. Gefährdungsbeurteilungen für mobile Tätigkeiten sind selten, Gefährdungsbeurteilungen für psychische Belastungen sind auch noch nicht die Regel. Die untersuchten Vereinbarungen weisen kaum Regelungen zum Gesundheitsschutz auf - und wo es Regelungen gibt, wird meist pauschal darauf verwiesen, dass die geltenden Bestimmungen zum Arbeits- und Gesundheitsschutz zu beachten seien. Mit solchen allgemeinen Regelungen wird die Verantwortung gewissermaßen umgekehrt, weil sie den Beschäftigten auferlegen, was Sache des Arbeitgebers ist. Auch die Nutzung mobiler Endgeräte wird kaum unter gesundheitlichen Fragestellungen geregelt. So werden ergonomische Aspekte von Smartphone, Tablet und Notebook weitgehend ausgeblendet. ${ }^{12}$

Fehlbelastungen können auch entstehen, wenn die technische Ausstattung nicht ausreicht oder nicht adäquat ist, der persönliche Umgang und Abgrenzungsmöglichkeiten bei theoretisch permanenter Erreichbarkeit nicht geübt, die Einbindung in Entscheidungen und der Informationsfluss mangelhaft sind, Termindichte und fehlende Mitsprache bei der Terminfindung herrschen. Diese Ergebnisse wie auch die jüngeren Ergebnisse des DGB-Index Gute Arbeit machen deutlich, dass der Arbeits- und Gesundheitsschutz bei mobiler Arbeit große Lücken aufweist. ${ }^{13}$ Differenzierte Gefährdungsbeurteilungen nach $₫ 5$ Arbeitsschutzgesetz (ArbSchG), die auch die besonderen Belastungen durch die Kombination aus Arbeits-, Reise- und Erreichbarkeitszeit mit einbeziehen, können der Ausgangspunkt für alle weiteren Überlegungen sein. Solche Gefährdungsbeurteilungen, die spezifische psychische Belastungen auch von mobiler Tätigkeit betrachten, verbreiten sich langsam, sie sind noch nicht die Regel. ${ }^{14}$

\section{Handlungsfeld Kompetenzentwicklung}

Mobile Arbeit erfordert von den Beschäftigten umfassende soziale und kommunikative Kompetenzen. Die Risiken eines mobilen Lebens mit seinen Unwägbarkeiten, Ungewissheiten und Anforderungen an die individuelle Beweglichkeit müssen gemeistert werden. Rahmenbedingungen ändern sich oftmals kurzfristig, sodass Entscheidungen zum Teil unter schwierigen Bedingungen schnell getroffen werden müssen.

Sich flexibel und schnell an veränderte Rahmenbedingungen mit richtigen Entscheidungen anzupassen, ist eine anspruchsvolle Aufgabe, die meist vorausgesetzt wird. Hinzu kommt, dass unter Bedingungen hoher räumlicher Mobilität die Fähigkeit relevant wird, Technologien zur Organisation beruflicher und sozialer Beziehungen einzusetzen und in sozialen und professionellen Netzwerken (Betrieb, Mitbestimmung, Freunde, Zivilgesellschaft etc.) eingebunden zu bleiben.

Die Diskussion um die Organisation und Arbeit von Unternehmen im Internetzeitalter (enterprise 2.0) ist noch nicht zu Ende, da läuft bereits die Debatte um Industrie-4.0.-Szenarien zur Arbeitsorganisation von morgen, die erahnen lassen, dass die Arbeitsformen und die Arbeitsorganisationen sich sehr verändern können. Dabei sind die Verbindungen aus digitalen Informationen, mobilen Endgeräten zur Informationsverarbeitung und ihre Integration in Produkte und Produktionsprozesse von entscheidender Bedeutung.

Aber kompetent zu sein im Umgang mit Mobilität und Digitalisierung, ist nicht selbstverständlich, sondern muss gefördert und entwickelt werden. Die Kompetenz, mit Medien umzugehen - in der Gegenwart noch Internet, Email, Social Media -, die Kompetenz, mit Mobilität umzugehen, d. h. fähig zu sein, an verschiedenen Orten, Räumen und Zeiten zu arbeiten, und die Kompetenz, sich selbst zu steuern (Zeit, Organisation, Disziplin, Konzentration), sind voraussetzungsvoll und nicht bei jedem Menschen gleichermaßen vorhanden. Die bloße technische Einweisung in die Nutzung mobiler

10 ebd.

11 Z. B. Prangert, B./Schüpbach, H. (2013): Die Auswirkungen arbeitsbezogener erweiterter Erreichbarkeit auf Life-Domain-Balance und Gesundheit, Dortmund.

12 Thannheiser, A. (2013): Mobile Endgeräte, Düsseldorf.

13 Bretschneider-Hagemes, M. (2011): Belastungen und Beanspruchungen bei mobiler IT-gestützter Arbeit, ZArbWiss, S. $223-233$.

14 Romahn, R. (2013): Gefährdungsbeurteilung, Frankfurt a. M. 
Endgeräte genügt nicht, um Medienkompetenz zu entwickeln. Und so bilden Verbote, die Beschäftigte vor zu langen Arbeitszeiten schützen, nur die Reißleine. Beschäftigte müssen selbst ihre Soll-Grenze ziehen können, ohne willkürliche Nachteile befürchten zu müssen. Das ist auch eine Frage der Unternehmenskultur.

Die Auswertungen der Vereinbarungen sowie der Fallstudien machen deutlich, dass im Bereich der Kompetenzentwicklung ein erhebliches Defizit besteht. Wie die Beschäftigten die Kompetenzen erwerben, das mobile und digitalisierte Leben zu meistern, vor allem Arbeitszeit, Datenschutz und Gesundheit im Blick zu behalten, wird ihnen selbst überlassen und damit individualisiert. Inzwischen entwickeln einzelne Unternehmen Bildungseinheiten für den Umgang mit Mobilität.

\section{Handlungsfeld Personalentwicklung}

Ein großes Defizit bei der Gestaltung mobiler Arbeit besteht im Bereich der Personalpolitik bzw. -entwicklung. In den Fallstudien wurde übereinstimmend von Betriebsräten und Personalverantwortlichen berichtet, dass es schwerer geworden sei, für hochmobile Tätigkeiten geeignetes Personal in ausreichendem Umfang zu finden. Verschärfend komme hinzu, dass insbesondere jüngere Beschäftigte häufig nicht mehr dauerhaft intensive Reisetätigkeiten ausüben wollen. Folge davon sei, dass Betriebe zunehmend Schwierigkeiten haben, Stellen zu besetzen, die permanent eine hohe Mobilitätsbereitschaft erfordern. Viele mobil Beschäftigte wollen nach einer Phase hoher Mobilität auch zeitweise oder auf Dauer weniger reisen. Vor dem Hintergrund dieser einhelligen Problembeschreibung überrascht es umso mehr, dass es in keinem der von uns untersuchten Betriebe eine darauf ausgerichtete Personalpolitik gab. Es fehlt an Ansätzen für eine betriebliche Personalpolitik und -entwicklung, die geänderten lebensweltlichen Anforderungen Rechnung trägt. Bislang werden weder gezielt Möglichkeiten geschaffen, den Umfang solcher Arbeit lebensalters- oder lebenssituationsbezogen zu gestalten, noch wird gezielt daran gearbeitet, weitere betriebliche Mitarbeiter dafür zu qualifizieren. Lediglich in einem Betrieb hat der Betriebsrat diese Frage von sich aus als Ziel seiner Arbeit thematisiert.

Hier benötigt die Interessenvertretung einen langen Atem und eine mittelfristige Strategie. Die Rechte bei der Personalplanung nach $\$ 92$ Betriebsverfassungsgesetz (BetrVG) geben zwar eine Diskussionsplattform, aber sie geben kaum einen unmittelbaren Gestaltungsansatz. Der Betriebsrat muss also überlegen, wie er klug und konstruktiv sein (meist noch ungenutztes) Mitbestimmungsrecht zu Versetzungen und Auswahlrichtlinien mit Arbeitszeitregelungen und Initiativen zur Personalplanung und -entwicklung und zur beruflichen Qualifizierung nutzt. Ein Rezeptbuch zum Erfolg gibt es dafür nicht.

\section{Was folgt daraus für die betriebliche Gestaltung?}

Wenn die Beschäftigten zunehmend eigenverantwortlich Unternehmensziele verfolgen, dann brauchen sie Autonomie in der Gestaltung und Kompetenzen im Umgang mit steigenden Mobilitätsanforderungen. Dazu benötigen sie weitgehende Freiräume bei der Organisation ihrer Arbeit, sodass sich passend für die jeweilige Lebenssituation ein zuträgliches Arrangement ergeben kann.

15 Carstensen, T. (2012): Grenzmanagement im Umgang mit dem Internet, in: Beyreuther, T./Duske, K./Eismann,Ch./Hornung, S./Kleemann, F.: Consumers@work, Frankfurt a. M., S. 180-192.
Für betriebliche Interessenvertretungen entsteht eine Zwickmühle: Einerseits besteht die Aufgabe darin, Beschäftigte vor Arbeitsverdichtung, zu viel Entgrenzung und überhöhten betrieblichen Flexibilitätsanforderungen zu schützen. Andererseits müssen jedoch auch wichtige Freiräume erhalten bleiben für die gewünschte individuelle Arbeitsorganisation und individuelle Zeitarrangements. Mobile Arbeit ist mit Freiheiten verbunden, die Beschäftigte nicht missen möchten. Selbst hilfreiche Regelungen werden nicht eingehalten, wenn sie als Bevormundung oder als Einengung von Freiheit interpretiert werden und damit das Gegenteil von dem bewirken, was eigentlich mit der Regelung bezweckt werden sollte.

Betriebliche Regelungen werden dann eher akzeptiert, wenn sie nicht an die Stelle individueller Lösungen treten, sondern zur Erweiterung von Handlungsspielräumen führen. Arbeitszeitregelungen, die die Arbeitszeit ausschließlich formal begrenzen, bewirken eher das Gegenteil: Erbrachte Arbeit wird nicht erfasst, bleibt unvergütet und ohne Ausgleich. So etwa bei Regelungen, die die Arbeitszeit auf maximal zehn Stunden begrenzen und eine Erfassung darüber hinausgehender Zeiten nicht erlauben. Der Monteur im Außeneinsatz wird dennoch länger arbeiten, wenn die Umstände es erfordern. Zeitkonten müssen auf- und wieder abgebaut werden können. Aber in der Praxis zeigt sich häufig, dass gut gemeinte kurze Ausgleichszeiträume dazu führen, dass der Ausgleich letztlich in Geld erfolgt.

Die Ambivalenz besteht fort: Durch technische Möglichkeiten moderner Kommunikation hat sich das Arbeitsleben beschleunigt und verdichtet. Für viele Beschäftigte wachsen individuelle Freiräume. Aber nicht für alle verbessern sich die Arbeitsbedingungen. Auch die Grenze zwischen Beruf und Privatleben verläuft nicht einheitlich gleich für alle Beschäftig ten, sondern erfordert unterschiedliches „Grenzmanagement “. ${ }^{15}$ Entsprechend sollten Regelungen ein Mindestmaß an Schutz geben und zugleich die Bedürfnisse von sehr unterschiedlichen Beschäftigtengruppen in den Blick nehmen. Darüber hinaus ist es die Pflicht des Arbeitgebers, Gefährdungsbeurteilungen durchzuführen und Gesetze einzuhalten. Dies zu kontrollieren, ist eine wichtige Aufgabe des Betriebsrates. Große Unternehmen gehen inzwischen dazu über, von der obersten Führungsebene eindeutige Signale auszusenden: Sie erwarten nicht, dass ihre Beschäftigten ständig erreichbar sind. Hinzu kommt die Eigenverantwortung von Beschäftigten, z. B. das Smartphone einfach mal auszuschalten.

\section{AUTOREN}

MANUELA MASCHKE, Dr., leitet das Archiv Betriebliche Vereinbarungen der Hans-Böckler-Stiftung.

manuela-maschke@boeckler.de

GERD NIES, Rechtsanwalt, war Vorstandsmitglied bei der IG Medien und ver.di.

gerd.nies@poste.it

GERLINDE VOGL, Dr., arbeitet als Soziologin in der Münchner Projektgruppe Sozialforschung. Arbeitsschwerpunkte: Mobilität und Arbeit.

gerlinde.vogl@sozialforschung.org 